\title{
Shedding light on the Negative Differential Resistance Effect observed in Organic Thin-Film Transistors
}

\author{
Authors: Eloi Ros1 , Marta Reig2 , Cristobal Voz1, Gintautas Bagdziunas3 , Pablo Ortega1 , \\ Dolors Velasco2, Joaquim Puigdollers1 \\ 1Universitat Politecnica de Catalunya, 2Universitat de Barcelona, 3Vilnius University
}

\begin{abstract}
Organic semiconductor electronics has the potential to become a solid and industrially feasible technology, with new and stimulating applications such as the flexible and transparent electronic devices. However, organic devices still have some drawbacks that must be solved in order to assure quality and reliability in complex electronic systems. In this work, we focus our attention to the physical origin involved in the Negative Differential Resistance phenomenon, often observed in the output electrical characteristics of organic thin-film transistors but rarely analysed.
\end{abstract}

Keywords Organic Field Effect Transistor, OFET, Negative differential resistance, Density of states.

\section{Introduction}

Field Effect Transistors (FETs) are one of the key device in modern electronics. Moreover, FETs based on thin-film semiconductors allow the fabrication of large-area Thin-Film Transistors (TFTs), opening a new fan-out opportunity of emerging applications [1]. Examples of this applications span from healthcare-monitoring devices, flexible displays, radio frequency identification tags, amongst other [2 - 5]. In particular, organic compounds are viable candidates for devices with such applications because they incorporate small molecules and conjugated polymers, which are cheap intrinsically soft and mechanically compatible with flexible substrates. Commercial electronics has been historically based on inorganic semiconductors such as Silicon, Gallium Arsenide, etc. Most of these inorganic FETs owe their position in the market place due to their high operating frequency and environmentally stable performance. Nevertheless, this has begun to change as a consequence of the low cost of organic compounds as well as the possibility to use solution-based processes and roll-to-roll manufacturing, which make organic technology an attractive cost effective industrial alternative. However, for OTFTs to be technologically viable they must achieve not only high electronic performance, but also reliable and comprehensible electrical characteristics. Experimentally, it is often observed that the electrical characteristics of 
OTFTs deviates from the ideal electrical characteristics [6]. These non-ideal behaviours (i.e., not consistent with standard models) lead to a variability on the resulting transistor parameters, making difficult the comparison of different devices and the establishment of reliable conclusions. Nonideal electrical characteristics usually found in OTFTs are, among others: contact effects, nonlinear transfer curve and the presence of high off-currents. The physical origin of these non-ideal effects are difficult to determine, but they are probably due to the presence of impurities (traps) in the bulk of the semiconductor and/or at the interface between the electrodes and the semiconductor, parasitic capacitances associated with overlap between the gate and the source/drain contacts, etcetera $[7-9]$.

One of the irregularities often observed when measuring OTFTs is the presence of Negative Resistance Effect (NDR) in the saturation region of the output characteristics, i.e. an increase in drain-source voltage $\left(V_{D S}\right)$ results in a decrease in drain-source current $\left(I_{D S}\right)$ for a fixed gate-source voltage $\left(V_{G S}\right)$ [10,11]. Understanding the origin of the NDR effect could provide guidelines for interpreting this particular non-ideal behaviour usually found in high mobility OTFTs, and provide new rules to tailor this phenomenon suiting the needs for specific applications.

Although a non-ideal current-voltage curve is an undesirable effect in the behavior of an OTFT, NDR effect possess by itself inherent interest, enabling the fabrication of devices with interesting applications, such as high-frequency oscillators [12], memory devices [13], and fast switches [14]. Therefore, there is a general effort to understand transport mechanisms that lead to it.

In this manuscript, we report the fabrication of a series of OTFTs based on tricyanovinylsubstituted carbazole-based materials that show a marked NDR effect in the saturation region of the output characteristics. The physical origin of the observed negative differential resistance is explained by taking into account the design of the transistor, the interaction between the $\mathrm{V}_{\mathrm{DS}}$ and $\mathrm{V}_{\mathrm{GS}}$ and the difference between the used organic molecules, mainly the different length of the Nalkyl chain of the carbazole moiety.

\section{Experimental}

The charge-transport properties of the compounds were studied in bottom-gate/top-contact OTFTs by using thermally grown $\mathrm{SiO}_{2}$ on crystalline silicon substrates and $\mathrm{Au}$ as source and drain electrodes (see Figure 1). The $\mathrm{SiO}_{2}$ dielectric surface (120 nm thick) was treated with polystyrene (PS) monolayer. Details of the PS monolayer fabrication can be found elsewhere [15]. All chemicals used to synthesize the compounds were of commercial grade and used as received. 9butyl-3-(1,2,2)-tricyanovinyl)-9H carbazole (compound 1 in the remainder of the article), (9-octyl3-(1,2,2)-tricyanovinyl)-9H-carbazole (compound 2), and (9-dodecyl-3-(1,2,2)-tricyanovinyl)$9 \mathrm{H}$-carbazole (compound 3 ) were prepared as previously reported $[1,15]$. 


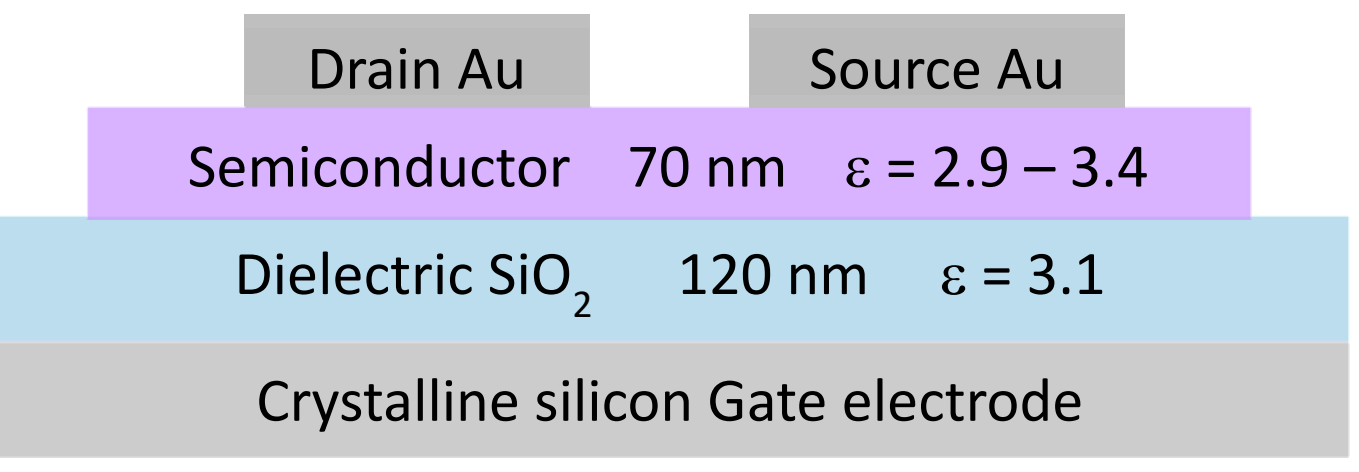

Figure 1

Scheme of the fabricated top-contact bottom-gate Thin-Film Transistor.

The organic compounds were deposited by the thermal vacuum evaporation technique (with a base pressure below $10^{-6}$ mbar). Sublimation temperature was regulated to maintain a stable deposition rate of about $0.1-0.2 \AA / s$ to obtain $75 \mathrm{~nm}$ thickness layer. Afterwards, samples were transferred to a different vacuum chamber to evaporate the metal contacts. Drain and source electrodes, were defined by means of a metallic shadow mask, with a channel length (L) and width (W) of $80 \mu \mathrm{m}$ and $2 \mathrm{~mm}$, respectively. The OTFTs were electrically characterized in the dark and under moderate vacuum conditions $\left(10^{-1} \mathrm{mbar}\right)$ by using a Keithley $2636 \mathrm{~A}$ source meter. The electron mobility $\left(\mu_{e}\right)$ and the threshold voltage $\left(V_{T}\right)$ were calculated in the saturated region and using the following equation (1):

$$
I_{D S}=\frac{W C_{o x} \mu_{e}}{2 \mathrm{~L}}\left(V_{G S}-V_{T}\right)^{2}
$$

where $W$ and $L$ are the channel width and length, respectively, and $C_{o x}$ is the capacitance of the dielectric per unit area.

\section{Operation principle of a Thin-Film Transistor (Accumulative channel vs Density-of-States).}

The geometry of a Thin-Film Transistor consists of a semiconductor layer deposited on top of a dielectric layer. The dielectric layer is in contact with a metallic electrode and constitutes the gate electrode. On the other hand, semiconductor layer is contacted with two electrodes that constitutes the drain and source contacts. In top-contact geometry, drain and source electrodes are deposited on top of the semiconductor layer, whereas bottom contact geometry drain and source are embedded on the semiconductor layer. In both cases, the conductive channel is in contact with the 
dielectric layer. The quality of the interface semiconductor/dielectric plays an important role on the device performance [16].

The usual way to explain the electrical output characteristic of the TFT is by means of the fieldeffect phenomenon. An application of a voltage on the gate electrode with respect of the source electrode $\left(V_{G S}\right)$ induce an accumulation of charge at the dielectric/semiconductor interface and electrical current is established when a transversal potential is applied via the drain/source electrodes $\left(V_{D S}\right)$. This accumulated charge modifies the channel resistance by many orders of magnitude upon the application of the $V_{G S}$ voltage. The amount of current that flows between the drain and source electrodes (IDS) upon the application of a voltage between the drain and source $\left(V_{D S}\right)$ depends on the resistance of the semiconductor channel. With no voltage applied to the gate, the resistance of the channel is very high and consequently, the current between drain and source is very low (this behaviour constitutes the off region of the transfer characteristics). The voltagecontrolled resistance of the channel is the essence of any field-effect transistor [17], and it is the usual way to interpret experimental results obtained with TFT based on organic semiconductors $[18,19]$.

However, in some cases, it is more appropriate to describe the working principles of a field-effect transistor using the energy levels of the different elements within the device. Energy levels of the source, drain and semiconductor are determined by their electrochemical potentials (i.e. Fermi level). In general Fermi levels or electrochemical levels are a measure of the potential energy, referenced to an energy axis, in which the lowest value of $0 \mathrm{eV}$ corresponds to the vacuum level (i.e. an electron at rest just outside the surface). Distribution of the electrochemical potential plays a central role when describing the current-voltage characteristics of a device from an energy approach [20,21]. In figure 2, we schematically plot the location of the electrochemical potentials of the main constituents of the TFT: source $(\mu s)$, drain $\left(\mu_{D}\right)$ and the semiconductor.

Electrochemical potentials $\mu_{\mathrm{s}}$ and $\mu_{\mathrm{D}}$ are related to the work function of the metals used as electrodes, i.e. the minimum energy necessary to remove one electron from the metal surface to its vacuum level position. Determination of the electrochemical potential of the semiconductor is, in general, a difficult task [22]. In the case of inorganic semiconductor, like crystalline silicon, the number of available electronic states in cubic $\mathrm{cm}$ per unit of energy (Density of States DOS) and the position of the electrochemical potential (Fermi level) with respect the conduction or valence band edge can be calculated with high level of exactitude. However, this is not the case in disordered semiconductors, in which the position of the electrochemical potential cannot be determined in a straightforward way. Electrochemical potential in organic semiconductor lies within the energy band gap and close to the High-Occupied Molecular Orbital (HOMO) or to the Low-Unoccupied Molecular Orbital (LUMO) levels depending on its ability on accepting or 
donating electrons. For our schematic description, the knowledge of the position of the electrochemical potential of the semiconductor is not important. For simplicity, we consider that source and drain electrodes are connected through an n-type semiconductor, in which the electrochemical potential is close to its HOMO level [23]. Same description applies for the case a p-type semiconductor with the electrochemical potential close to the LUMO level.

$$
q V_{D S}=\mu_{D}-\mu_{S} \quad q V_{G S}=\mu_{G}-\mu_{S}
$$
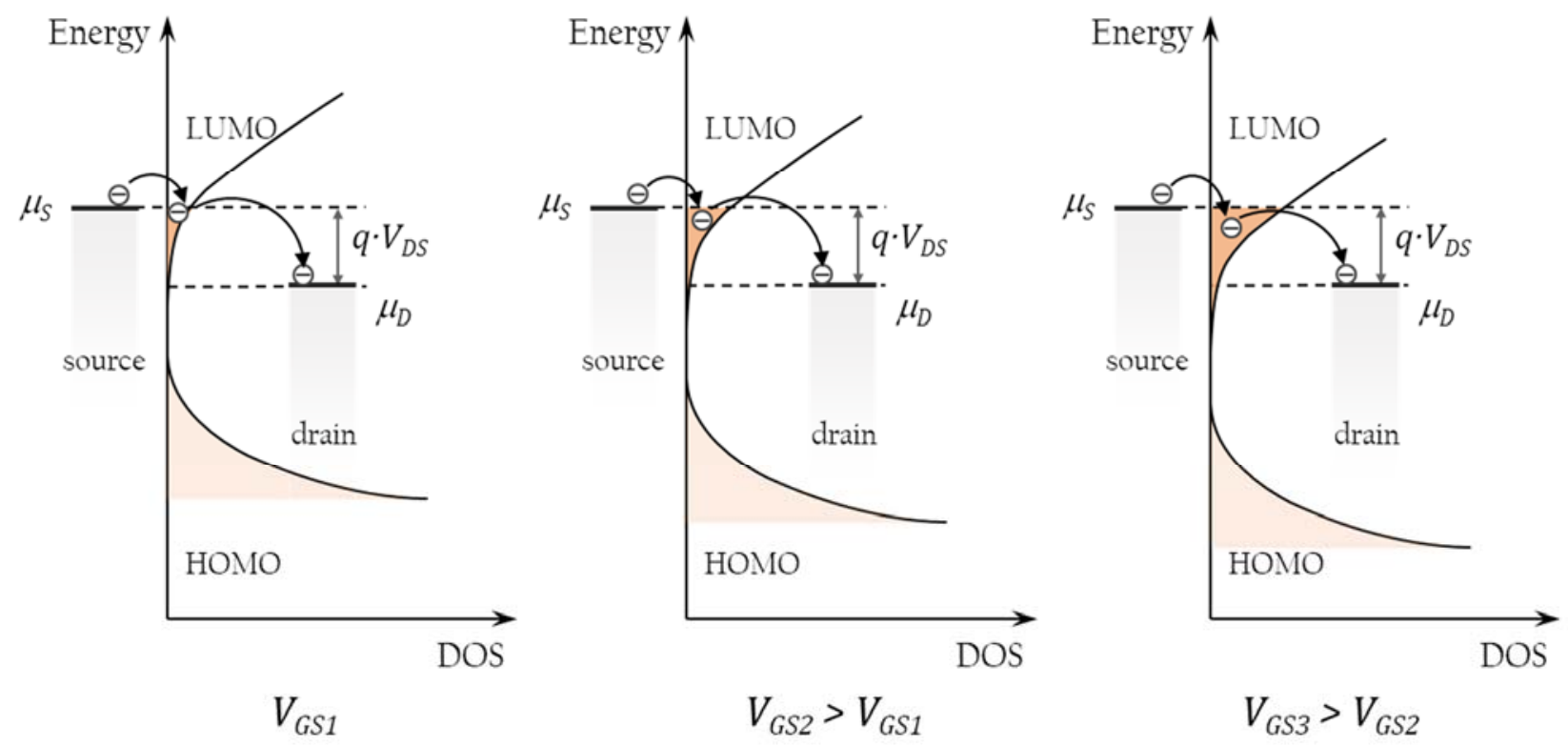

Figure 2.

Energy band diagram corresponding to the transfer characteristics in a n-type transistor. $\mu_{D}, \mu_{S}$ and $\mu_{G}$ are the electrochemical potential of the drain, source and gate electrodes respectively.

The number of band-tail available electronic states available to conduction is highlighted in dark orange colour.

At equilibrium, all electrochemical potentials are at the same energy level everywhere. At absolute zero temperature all electronic states with energy $E<\mu$ are filled with electrons while all sates with $E>\mu$ are empty. At any temperature the transition from completely empty or filled states occurs over an energy of $+/-2 k T$ around $E=\mu$, where $k$ is the Boltzmann constant and $T$ is the absolute temperature. This transition is described by using the Fermi function [20]. 
Application of positive voltage to the drain electrode with respect to the source electrode (chosen as a reference) splits source and drain electrochemical potentials, being drain level at high-energy position and source at low-energy position (Figure 3 left).
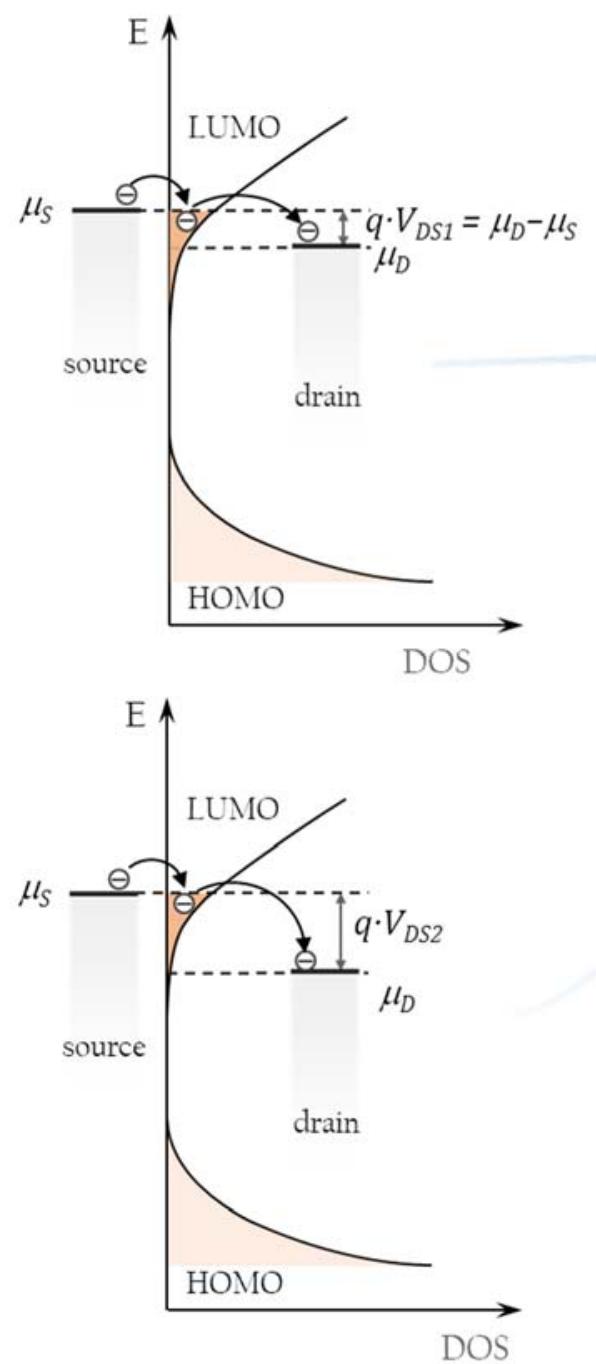
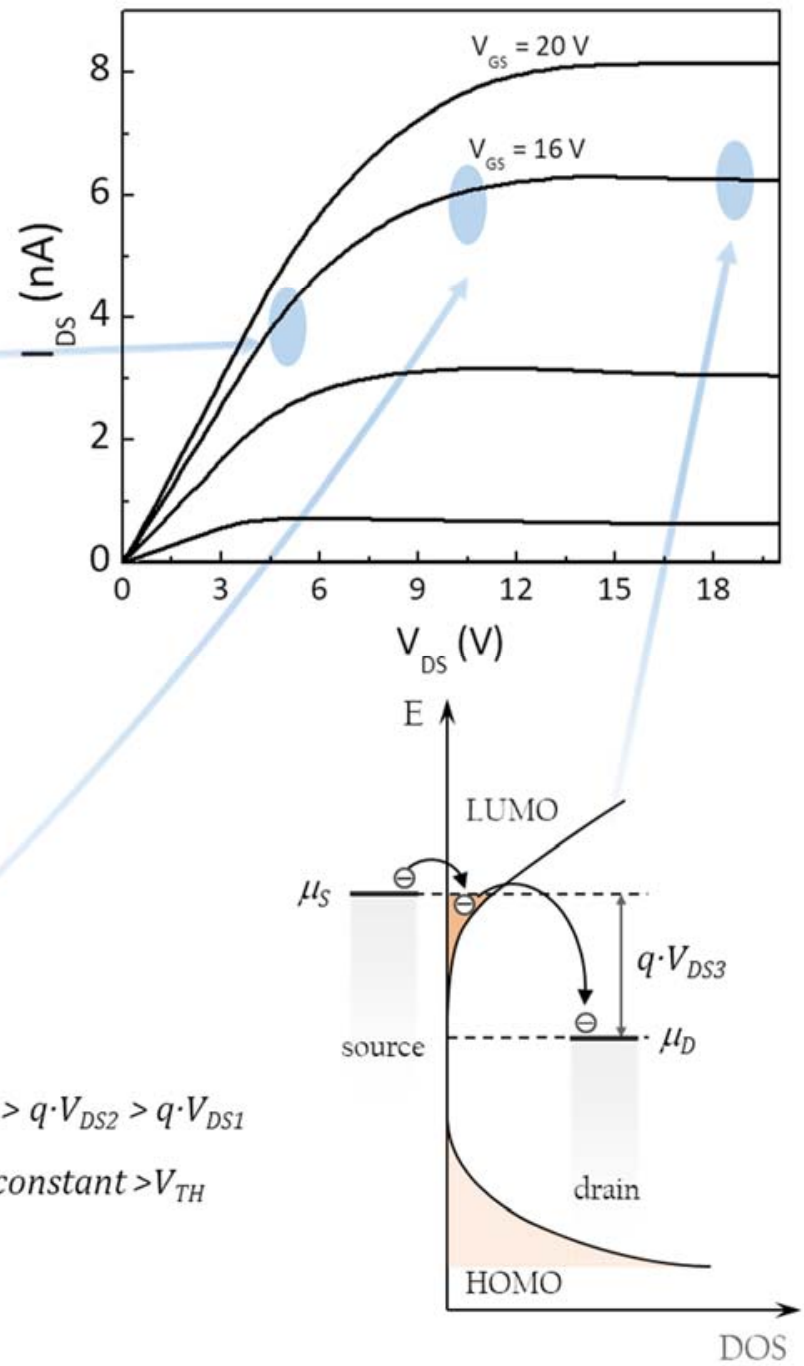

Figure 3.

Energy band diagram in the output characteristics in an n-type transistor. $\mu_{D}$ and $\mu_{S}$ are the electrochemical potential of the drain and source electrodes. The number of band-tail available electronic states for conduction is highlighted in dark orange colour. 
Now, the system is forced into a non-equilibrium state. To balance back to equilibrium the contacts energy levels, electrons from source flow to lower energy states of the drain electrode through electronic states located in the n-type semiconductor. Since external voltage maintains the drain electrode at low energy the system is kept outside of its equilibrium condition, causing electrons to flow continually from source to drain electrode. Current between source and drain is proportional to the carrier density $n$ of electrons on the conduction channel, being the carrier density proportional to the product of the density of electronic states and the Fermi-Dirac probability function (Fermi function). The higher the number of available electronic states at the semiconductor the higher would be the number of electrons flowing from source to drain (i.e. higher drain-source current). However, current is zero if the material does not have electronic states are available between $\mu_{\mathrm{s}}$ and $\mu_{\mathrm{D}}$, regardless of the external applied voltage.

Let us examine the situation when a positive voltage is applied to the gate electrode with respect to the source, as schematically is represented in Figure 2 (left $V_{G S}=V_{G S 1}$, center $V_{G S}=V_{G S 2}>V_{G S 1}$, right $V_{G S}=V_{G S 3}>V_{G S 2}$ ). We consider source - drain voltage $V_{D S}$ positive and constant in value. The application of a gate voltage $\left(V_{G S}\right)$ changes the potential in the channel. A positive $V_{G S}$ lower all electronic states down in energy. Increasing $V_{G S}$ values shift more downwards (with respect the energy axis) the electronic states of the semiconductor with respect the electrochemical potentials of the source and drain that remains unaffected, since $V_{D S}$ has not changed. A window of available states appears between $\mu_{\mathrm{s}}$ and $\mu_{\mathrm{D}}$, as shown in Figure 2, and electrons flows from source to drain, trying to drive the system into equilibrium. Current is established between source and drain, being proportional to the number of energy levels available between the electrodes electrochemical potentials $\mu_{S}-\mu_{D}$.

In summary, application of a $V_{G S}$ results in an increase of available electronic states, making the channel more conductive, and eventually increasing IDS. This electronic behaviour corresponds to the transfer characteristic of the transistor. Threshold gate voltage $\left(V_{T}\right)$ is the voltage needed to turn the transistor on and is determined by the energy difference between the equilibrium electrochemical potential of the source and drain $\left(\mu_{S}=\mu_{D}\right)$ and the lowest available empty electronic state of the semiconductor, that in the case of n-type semiconductor corresponds to the conduction LUMO edge. The energy difference between the electrochemical potentials of the electrodes and of the semiconductor can be adjusted by the introduction of an electric field at the interface dielectric/semiconductor. This is usually performed by the introduction of permanent dipoles at the interface. One of the most successful methods to do this is by using Self-Assembled Monolayers that incorporate a permanent dipolar moment. 
In our description, we have considered a DOS that is constant all along the conductive channel between source and drain electrode. However, in a real case, potential distribute along the conduction channel, modifying the distribution of DOS, being the number of available states higher close to the source electrode and lower close to the drain electrode.

From the previous description, it is clear that a detailed analysis of the transfer characteristic of an OTFT provide valuable information about the distribution of localized states on the vicinity of the electrochemical potential (Fermi level) of the semiconductor. Higher disorder in the thin-film semiconductor results on higher LUMO (HOMO) broadening distribution of states, with tail states extending further in the bandgap and the possible existence of deep states. All these states contribute to the current, although LUMO (HOMO) conduction edge could lie outside the energy window defined by $\mu_{s}-\mu_{\text {D }}$. Electrical current value on the off region of the transfer characteristics depends to the distribution of trap states located in the mid-gap of the semiconductor (deep states). Sub-threshold region current (below $V_{T}$ ) relates to the number of localized tail states, whereas linear region (higher $V_{G S}$ ) is proportional to the number of states on the LUMO (HOMO) conduction edge. In this picture, it is clear that knowing the energy dependent distribution of DOS in the semiconductor helps to understand the electrical behaviour of the Thin-Film Transistor. Furthermore, spectral energy DOS distribution influences not only the current flowing between drain and source electrodes, but also the threshold voltage and subthreshold shape of the OTFT. [24]

This model becomes easier to implement on well-ordered semiconductors, like single-crystal compounds, with delocalized electronic states forming a Conduction Band (Valence Band for ptype semiconductors) or with a well-defined Valence and Conduction Band onsets. However, for disordered compounds like polymers, polycrystalline and amorphous small molecule semiconductors, the distribution of energetic states around the LUMO level (HOMO for p-type semiconductors) does not have a well-defined onset, and the distribution of localized electronic states extends into the energy band gap. The broadening of the HOMO (LUMO) levels, due to the energetic material disorder, is described by adding a distribution of tail states that act as traps. An exact distribution of these tail states is still missing in organic semiconductors and different approaches are used to sketch its shape. Sometimes it is used an exponential distribution [23], including deep and band tail states, as in the case of the inorganic semiconductor hydrogenated amorphous silicon. Other approach, more common in the field of organic semiconductors field, consist on approximate these tail states as a Gaussian distribution with typical widths of the order of $0.1-0.2 \mathrm{eV}$. An excellent discussion on the different approaches used to describe the energy distribution of states in organic semiconductors can be found in ref. [23]. In our case, knowing the 
exact DOS shape is irrelevant for the discussion, and a Gaussian distribution tail states is choose for visualization purposes.

\section{Description of the output characteristics.}

In the previous paragraphs, we have described the transfer electrical characteristics of a TFT considering the electrochemical potential of the contacts and the DOS of the semiconductor. A similar approach can be used to describe the output characteristics of the TFT. In Figure 3 shows a typical output electrical characteristics measured in an n-type semiconductor. Let focus on the variation of $I_{D S}$ as a function of $V_{D S}$ for a fixed $V_{G S}$. As previously discussed, current between source and drain is proportional to the number of available electronic states between $\mu s-\mu D$. Application of $V_{D S}$ voltage splits away $\mu_{s}$ and $\mu_{D}$, increasing the number of available electronic sates at the semiconductor and, consequently, increasing the number of electrons flowing from source to drain. Once the voltage $V_{D S}$ increases up to certain value $I_{D S}$ current saturates. The reason of this saturation is explained in Figure 3. When the electrochemical potential of the drain lies below the band edge of the LUMO, the current does not increase any more since the number of available states at the semiconductor remain fixed.

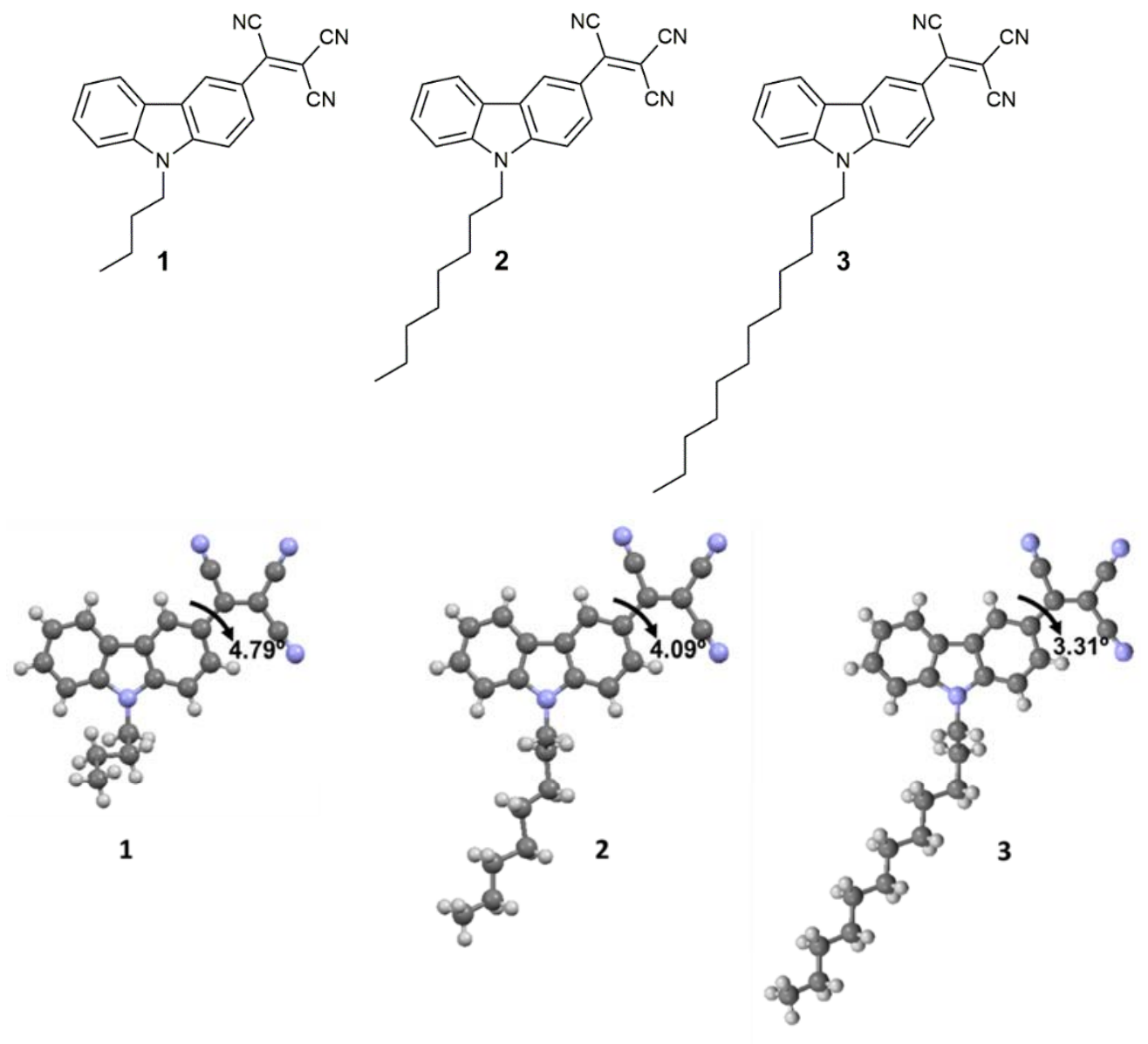

Figure 4.

Molecular structure of compounds (1), (2) and (3). 


\section{Results}

Focusing now on the devices we have fabricated, let us to introduce the differences between the used organic compounds. The main difference between the three compounds is the number of carbon atoms contained in the aliphatic chain of the molecule (Figure 4). Therefore, the length of the $\mathrm{N}$-alkyl chain attached to the carbazole moiety is the changing factor for the three compounds. Most of the organic compounds owe its electronic properties to the intra/intermolecular delocalized $\pi$ bonding. In the case of a small molecule semiconductor, the importance of the intermolecular $\pi$ bonding becomes clear. Molecular packing determines the electronic properties of the device as a direct consequence of the spatial distribution of the delocalized electronic bonds of the organic layer.
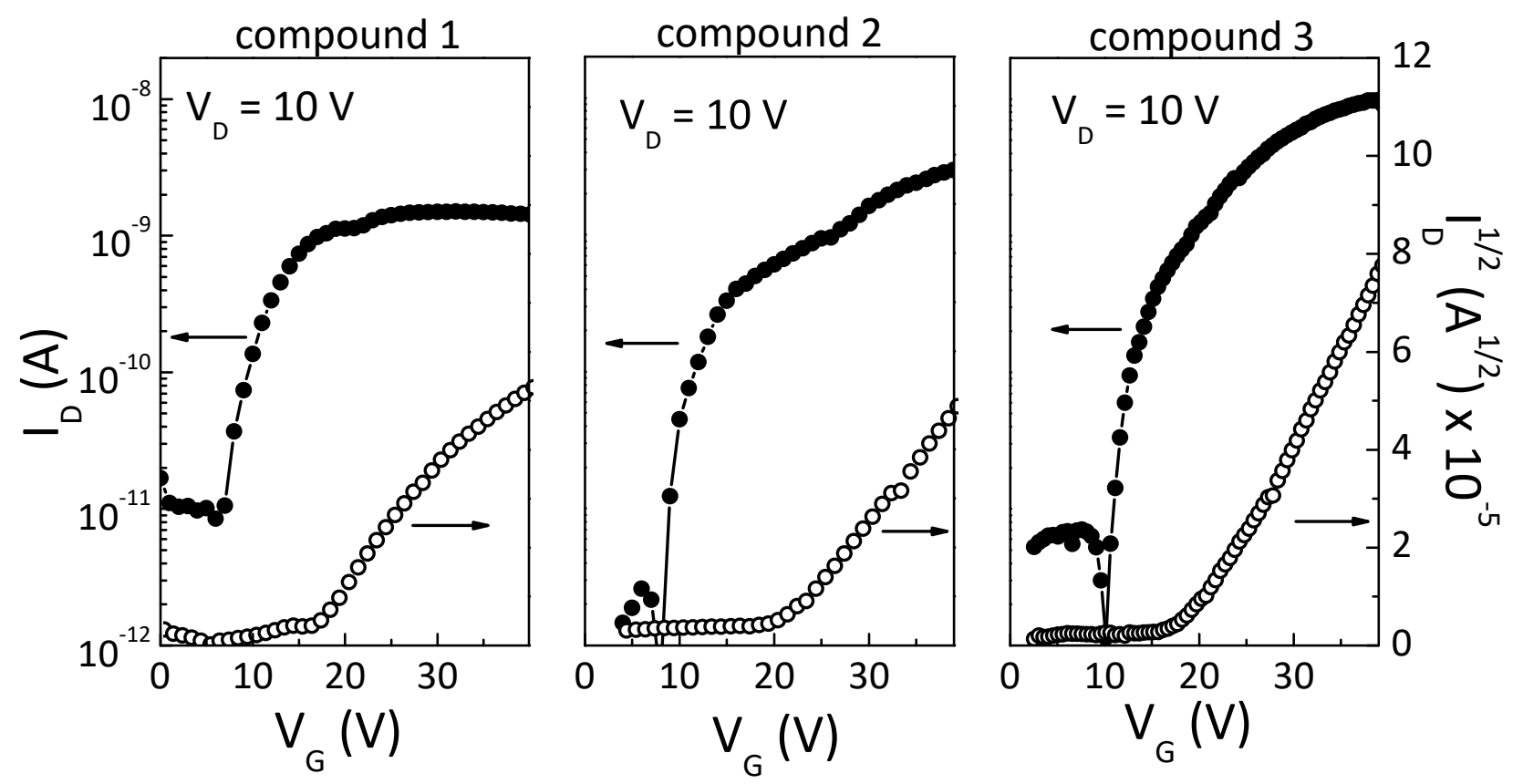

Figure 5. Transfer (left-axis) and saturation (righ-axis) characteristics for the compounds (1), (2) and (3).

Figure 5 shows the measured transfer and saturation characteristics of the OTFTs fabricated with the three compounds. All the devices shown typical electrical behaviour of n-type transistor. The main electrical parameters characterizing the TFT: carrier mobility, threshold voltage and Ion/Ioff ratio are summarized in Table 1. All the compounds exhibited similar electron mobility, extracted from the saturation characteristics, in the range of $2 \cdot 10^{-5}$ to $5 \cdot 10^{-5} \mathrm{~cm}^{2} \mathrm{~V}^{-1} \mathrm{~s}^{-1}$. Threshold voltages were also similar for all the prepared devices $(15.3-19.6 \mathrm{~V})$. The $\mathrm{N}$-alkyl chain length of the carbazole moiety has a negligible effect on the charge mobility. The similarities are according to the comparable antiparallel arrangement in the $\pi$ stacks with analogue interlayer distances that all of them exhibit. As reported previously [15], X-Ray Difraction analysis revealed that all 
compounds show a layered organization with an antiparallel arrangement and similar minimum interlayer distances (3.35-3.40 $\AA$ ), independently of the length of the N-alkyl chain.

Table 1. OTFT characteristics of devices based on tricyanovinyl-substituted carbazole derivatives 1-3 with PS-treated crystalline silicon/SiO 2 substrates.

\begin{tabular}{cccc}
\hline Compound & $\begin{array}{c}\boldsymbol{\mu}_{\boldsymbol{e}}{ }^{\mathrm{a}} \\
\left(\mathbf{c m}^{\mathbf{2}} \mathbf{V}^{-\mathbf{1}} \mathbf{s}^{-1}\right)\end{array}$ & $\begin{array}{c}\boldsymbol{V}_{T}{ }^{\mathbf{b}} \\
(\mathrm{V})\end{array}$ & $\begin{array}{c}\boldsymbol{I}_{\text {on }} / \mathbf{I}_{\text {off }}{ }^{\mathrm{c}} \\
(\mathrm{A} / \mathrm{A})\end{array}$ \\
\hline $\mathbf{1}$ & $2 \times 10^{-5}$ & 15.3 & $\sim 10^{2}$ \\
$\mathbf{2}$ & $3 \times 10^{-5}$ & 19.6 & $\sim 10^{4}$ \\
$\mathbf{3}$ & $5 \times 10^{-5}$ & 19.6 & $\sim 10^{4}$ \\
\hline
\end{tabular}

${ }^{a}$ Electron mobility $\left(\mu_{e}\right) .{ }^{b}$ Threshold voltage $\left(V_{T}\right) .{ }^{c} I_{o n} / I_{\text {off }}$ ratio. OTFT measurements were determined in vacuum and at room temperature.

However, a more accurate analysis show that devices based on the derivatives with the longest alkyl chains (compound 3), presented higher on current and larger $I_{o n} / I_{\text {off }}$ ratios $\left(\sim 10^{4}\right)$. The improvement of the $I_{\text {on }} / I_{\text {off }}$ ratio could be related to the different molecular packing motif and degree of organization of the molecules in the film. Molecules with the shorter butyl chain (compound 1) adopt a herringbone structure, whereas molecules with the longer octyl and dodecyl chains (compounds 2 and 3, respectively) adopt a coplanar arrangement with the $\pi$ stacks separated trough the interdigitated long alkyl chains as seen in Figure 7. This kind of solid-state organization induces a higher degree of long range organization of the molecules in the films as shown previously by Grazing Incidence X-ray Diffraction (GIXRD) studies [15].
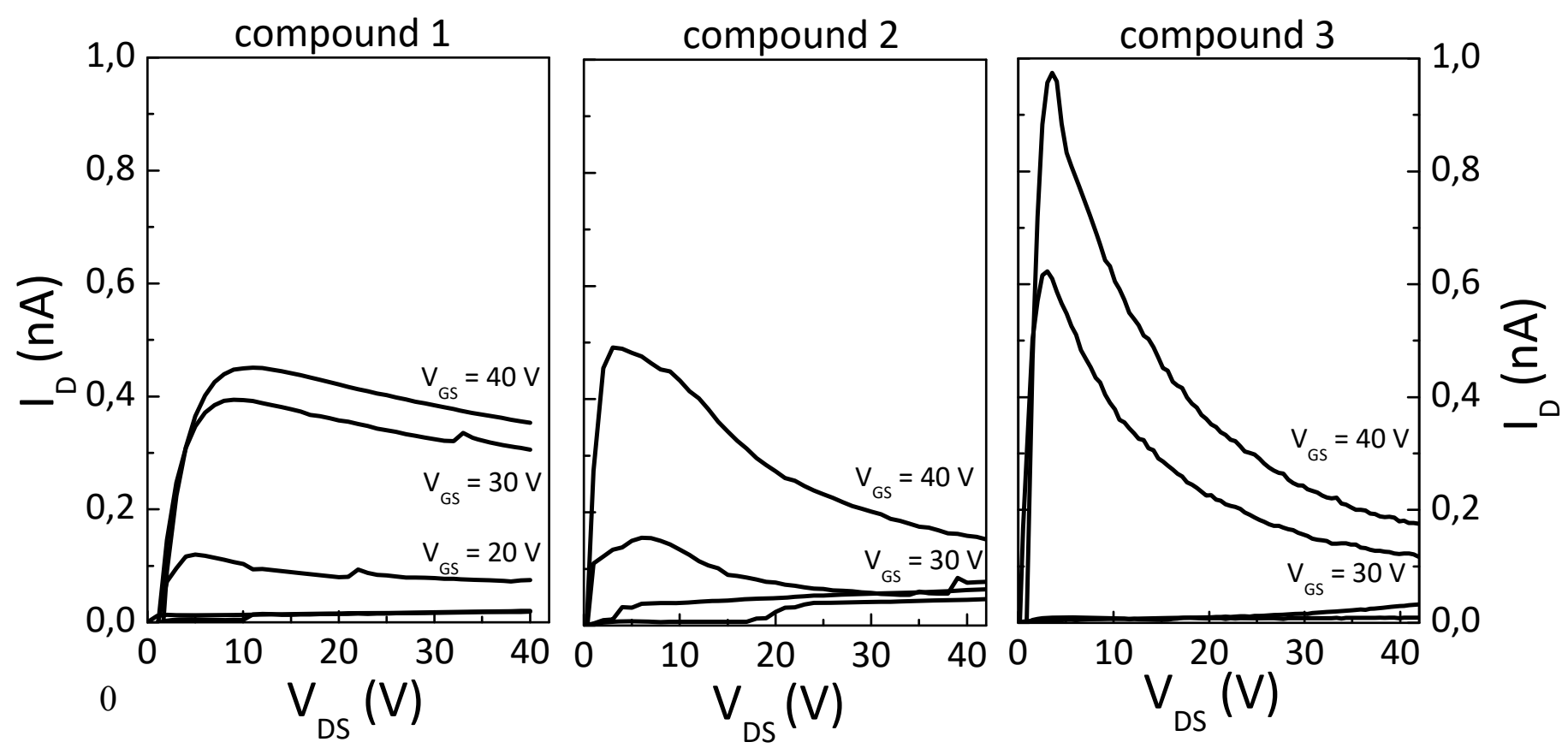

Figure 6.

Output chracateristics corresponding to compounds (1), (2) and (3). 
Figure 6 shows the output characteristic corresponding to the three compounds. For low $V_{D S}$ the current scales linearly with the applied voltage, without the presence of the crowding effect, indicating good carrier injection at the electrodes. For higher $V_{D S}$ values a saturation of $I_{D S}$ would be expected, however a decrease of the current with the voltage is observed. This NDR behaviour occurred for all the devices, being more pronounced for devices based on compound 3 .

It is important to point out that our TFT structure is quite symmetrical from the point of view of both, the active and the dielectric layer. Semiconductor and dielectric layer have similar thicknesses and permittivity values, making the TFT structure symmetrical from the channel point of view with respect the drain and gate electrode. In Table 2 we show the static dielectric constants $\varepsilon$ (permittivity) of compounds 1, 2 and 3 estimated according to the Clausius-Mossotti equation (S1). Permittivity values are of the same order of magnitude of the silicon dioxide used as a dielectric in the TFT. Permittivity values of the three compounds, although lying on the same order of magnitude, shows a slight change with the length of the alkyl functional group attached to the carbazole core unit [25]. As expected, the increase of the length of the aliphatic chain (i.e. apolar part of the designed molecules) implies a decrease of the permittivity in the resulting thin film. Interestingly, compound 3 which showed the a markedly NDR effect in the output characteristics has the lowest permittivity value. In addition, it can be observed how the degree of NDR effect measured on the devices scales inversely with the permittivity value. It is also worthy to note that the long interdigitated alkyl chains in compounds 2 and 3 (Figure 7) act as insulating parts but favour the formation of better organized films, which could be related to the improvement of the OTFT device performance as mentioned above [15].
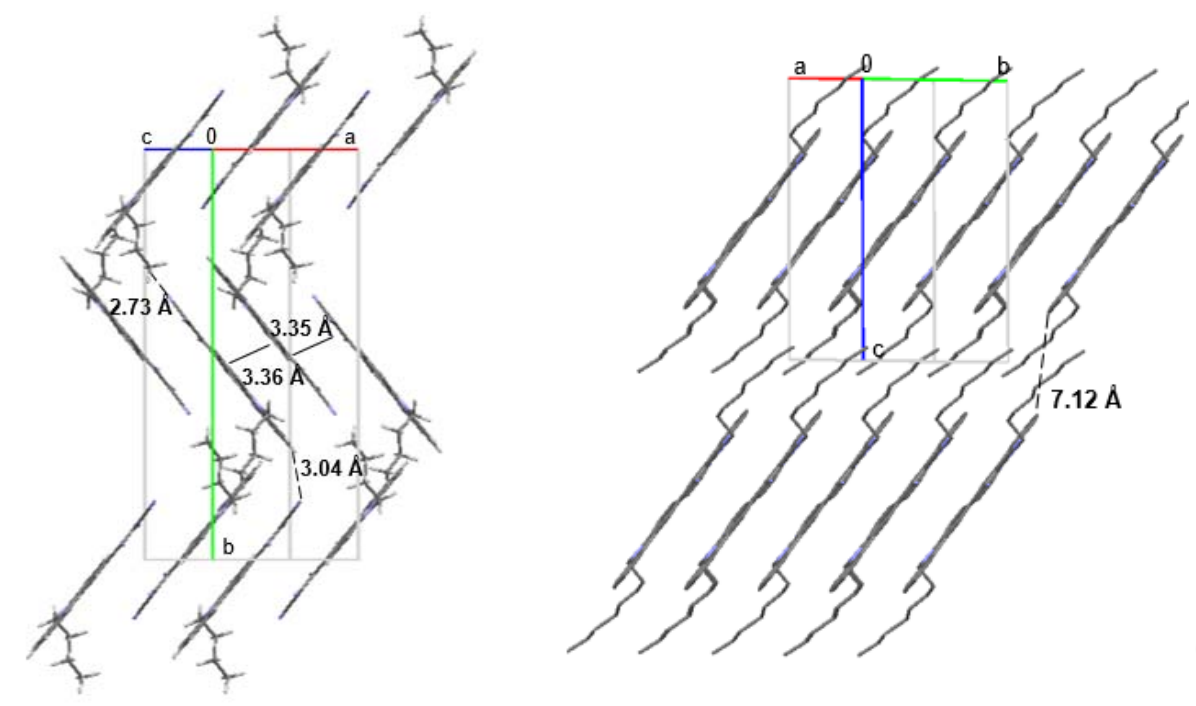

Figure 7.

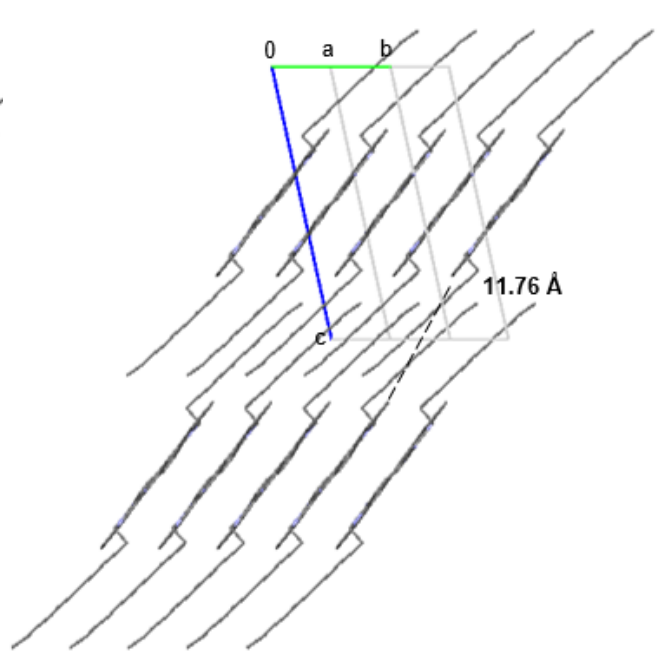

Crystal structures of compounds (1), (2) and (3). Hydrogen atoms have been removed for clarity in the representation of the molecular packing. (See Reference [12] for more detailed 
From this particular situation, one can interfere that there is some kind of correlation between the permittivity value of the compounds and the NDR effect. From previous observations, we believe that the application of a VDS not only splits the Fermi levels from the source and drain electrodes, but due to the existing symmetry in the geometry of the device, the drain source voltage also displaces the DOS of the semiconductor upwards with respect to the energy axis. This displacement reduces the number of available conduction states and, consequently, decreases the IDS current, as observed by the NDR in the output characteristics of the device. This effect is more pronounced in the saturation region, when the pinch-off of the channel has already occurred. This observation can be understood, as a screening effect of a large drain-source voltage over the electric field generated by the gate-source voltage near the drain contact, thus decreasing the carrier density in the channel near the drain contact and therefore reducing the number of carriers.

In summary, in this paper we show that the transport mechanism behind the NDR effect depends on the number of electronic states available for conduction in the semiconductor. This number of states is determined by the properties of the material (in particular in the dielectric/semiconductor interface) and the distribution of the energy bands in the device. The position of the energy levels results from the distribution of electric field, which in turn has a direct relationship with the geometry and permittivity of the different elements that constitute the device. From the point of view of the design of the organic semiconductors, small variations at the molecular level are shown here to be directly reflected in the macroscopic properties of the material, specifically in the permittivity, which at the same time exerts an influence on the operability characteristics of the transistor, through the so-called NDR effect. As a result, we can affirm that from the use of semiconductor compounds with specific permittivity values together with the use of optimized device geometry for the distribution of electric field, a control of the NDR effect is possible. The greater control in the synthesis of compounds (including 2D semiconductors) and the development of processing technologies at the nanometric scale opens up the possibility of manufacturing devices that exploit the technological applications offered by the NDR effect.

Table 2. Computed static dielectric constants, molecular volumes (with Van der Waals radius of $1 \AA)$ and static isotropic polarizabilities.

\begin{tabular}{cccc}
\hline Comp. & Volume $/ \AA^{\mathbf{3}}$ & Polarizability $/ \AA^{\mathbf{3}}$ & $\boldsymbol{\varepsilon}$ \\
\hline $\mathbf{1}$ & 347.2 & 68.85 & 3.43 \\
\hline $\mathbf{2}$ & 421.1 & 74.85 & 3.13 \\
\hline $\mathbf{3}$ & 494.9 & 80.84 & 2.93 \\
\hline
\end{tabular}




\section{Analytic model for a Negative Differential Resistance Transistor}

In order to explore whether the interaction between $V_{D S}$ and $V_{G S}$ is the true cause for NDR, we have developed a mathematical model including this interaction that resolves in a simple equation. We will assume that for the most part the current in the transistor output will be the drift current in the channel, and therefore it can be described as:

$$
\begin{gathered}
I=\int_{\mathrm{y}} \int_{\mathrm{z}} J_{x} d z d y=\int_{0}^{W} \int_{0}^{t_{s c}} \sigma E_{x} d z d y=\int_{0}^{W} \int_{0}^{t_{s c}} e \mu_{n} n(y) E_{x} d z d y= \\
\mathrm{W} e \mu_{n} E_{x} \int_{0}^{t_{s c}} n(y) d y=\mathrm{W} \mu_{n} \frac{d V_{c h}}{d x} \int_{0}^{t_{s c}} e n(y) d y
\end{gathered}
$$

Where $\mu_{n}$ is the mobility, $V_{c h}$ is the potential in the channel, $\mathrm{W}$ is the width of the contact, and $n(y)$ is the carrier density in the channel. The drift current density will be expressed with the Electric field in the channel (generated by VDS) times the conductivity. Conductivity in the channel itself depends on the amount of mobile charge present (controlled by $\mathrm{V}_{\mathrm{GS}}$ ) as well as other parameters such as the mobility. Eventually this resolves to equation (2) for the current.

$$
\mathrm{I}=\mathrm{W} \mu_{n} \frac{d V_{c h}}{d x} Q_{n}
$$

The charge present in the channel will be determined by the capacitor (Field Effect charge), and therefore can be summarized using following equation [26]:

$$
Q_{n}=C_{o x}\left[\left(V_{G S}-V_{c h}(x)-V_{T}\right)\right]
$$

With this expression for the charge in the channel we can introduce equation (3) into equation (2) and integrate to resolve for the current:

$$
\int_{0}^{L} I d x=\mathrm{W} \mu_{n} \int_{0}^{L} \frac{d V_{c h}}{d x} Q_{n} d x=W \mu_{n} C_{o x} \int_{0}^{L} \frac{d V_{c h}}{d x}\left(V_{G S}-V_{x}-V_{T}\right) d x
$$


Since the integral becomes independent of $\mathrm{x}$, one obtains the following expression:

$$
I=\frac{W}{L} \mu_{n} C_{o x} \int_{\mathrm{V}_{\mathrm{ch}}(0)}^{V_{c h}(L)}\left(V_{G S}-V_{c h}-V_{T}\right) d V_{c h}
$$

Now this becomes the classical output of a MOSFET once one integrates over $V_{D S}$ (i.e. $V_{c h}(0)=$ $V_{S}$ and $\left.\mathrm{V}_{\mathrm{ch}}(L)=V_{D}\right)$. Nevertheless, the geometric configuration and dielectric properties in organic semiconductors allows us to assume that there are other effects constraining this assumption. For example, in top contact configuration the electrodes are placed across the organic semiconductor layer and therefore there is a certain space between the charge in the channel (controlled by $\mathrm{V}_{\mathrm{GS}}$ ) and the Drain Source electrodes. This could allow multiple interaction effects of between the applied $V_{D S}$ and $V_{G S}$.

We will simplify all these multiple interactions in first order as a linear function proportional to the current (i.e. $\mathrm{V}_{\mathrm{ch}}(0)=V_{S}+r_{N D R} \cdot I$ and $\mathrm{V}_{\mathrm{ch}}(L)=V_{D}-r_{N D R} \cdot I$ ). This assumption originates from the proportional character of the charge in the channel (i.e. DOS energetic location) with the total intensity. For simplicity we can understand this as a factor $\Delta V=r_{N D R} I$ that is the main interaction potential. Now the integral can be written as equation (6):

$$
I=\frac{W}{L} \mu_{n} C_{o x} \int_{V_{S}+\Delta V}^{V_{D}-\Delta V}\left(V_{G S}-V_{c h}-V_{T}\right) d V_{c h}
$$

Solving the integral yields an implicit equation (7) which after resolving for the current becomes a compact expression seen in equation (8).

$$
\begin{gathered}
\left.L I=W \mu_{n} C_{o x} \mathrm{~L}\left(V_{G S}-V_{T}\right) V_{D S}-V_{D S}^{2} / 2\right\rfloor-W \mu_{n} C_{o x} r_{N D R} I\left(2\left(V_{G S}-V_{T}\right)-V_{D S}\right) \\
I_{D}=\frac{\frac{W}{\mathrm{~L}} \mu_{n} C_{o x}\left[\left(V_{G S}-V_{T}\right) V_{D S}-\frac{V_{D S}^{2}}{2}\right]}{1+\frac{W}{L} \mu_{n} C_{o x} r_{N D R}\left[V_{D S}-2\left(V_{G S}-V_{T}\right)\right]}
\end{gathered}
$$

The obtained expression has a hyperbolic term added to the classical MOSFET output that is accountable for the NDR effect. Furthermore, due the pinch off of the channel the equation in saturation becomes further accentuated by the NDR term which is also observed in the experimental output. 


$$
I_{D}=\frac{\frac{W}{\mathrm{~L}} \mu_{n} C_{o x}\left[\frac{\left(V_{G S}-V_{T}\right)^{2}}{2}\right]}{1+\frac{W}{L} \mu_{n} C_{o x} r_{N D R}\left[V_{D S}-2\left(V_{G S}-V_{T}\right)\right]}
$$

Using the corresponding parameters to the fabricated devices we can find the values of $r_{N D R}$ that results in a perfect fitting of the output characteristics obtained as seen in Figure 8. The resulting values obtained are $9 M \Omega, 44 M \Omega, 45 M \Omega$. Seems that due to the hyperbolic behaviour of this effect the output becomes very sensitive to the value of the parameter near the singularity of the equation. One can try to implement higher order terms in the definition of the interaction potential (i.e. $\left.\Delta V=r_{N D R} I+O\left(I^{2}, I^{3} \ldots\right)\right)$ which could provide complementary information to $r_{N D R}$ and resolve the problem of the singularity, nevertheless using this approach may require numerical solving. In the case of the tricyanovinyl-substituted carbazole OTFT it seems obvious that there is a correlation between the length of the alkyl chain and the value of the $r_{N D R}$. This could be due to either anisotropy in conductivity (related to the structure and shape of the molecule), or since the length of the chain is also correlated with the dielectric properties of the organic semiconductor, dielectric permittivity could also be an important factor involved in the degree of negative differential resistance observed. An enhanced control over the NDR in a transistor could provide lots solutions to applications in need of a device that can display an OFF/ON/OFF state without varying the Gate potential.
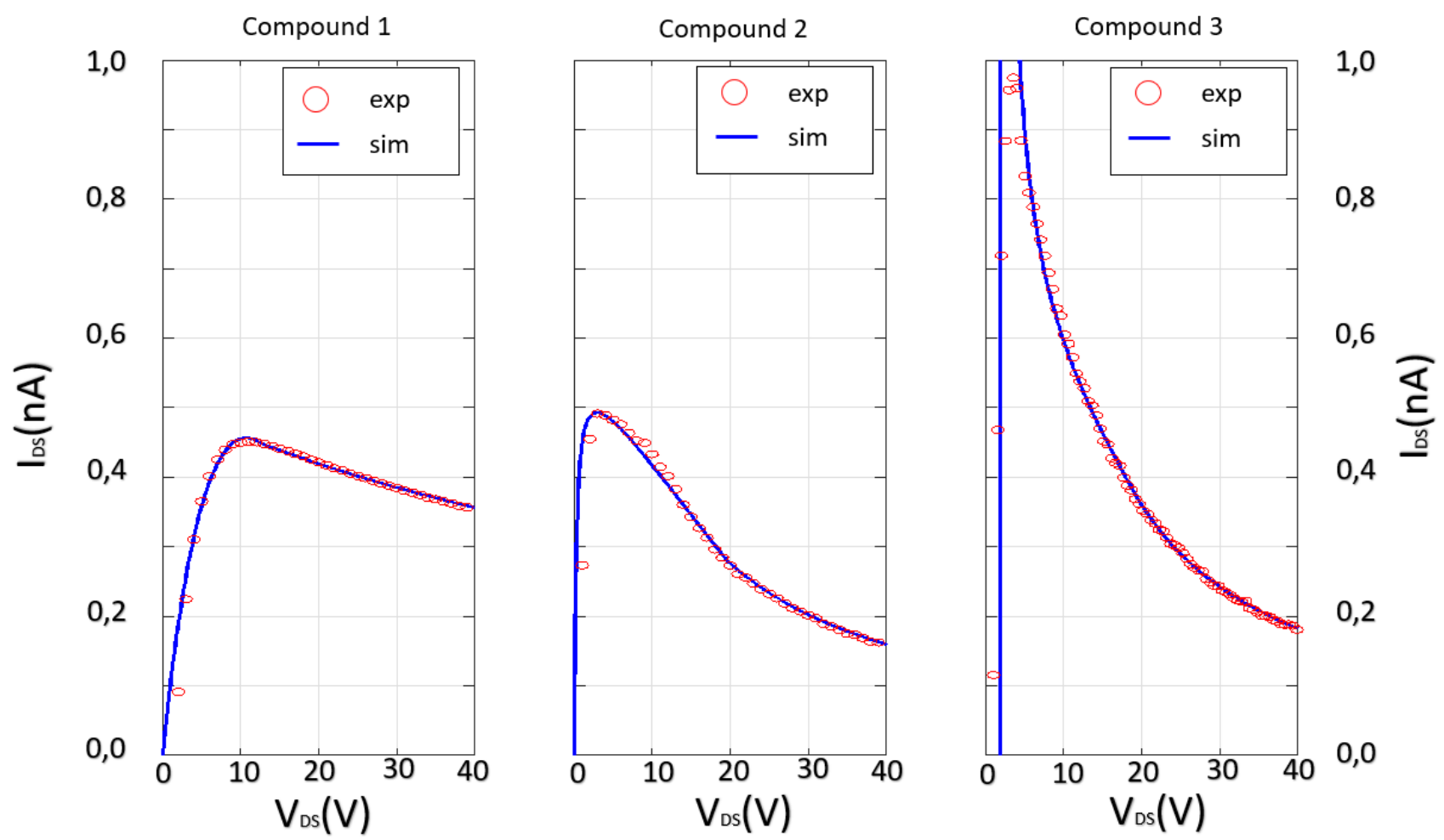

Figure(8) Output obtained from the model equations compared to experimental results for the different compounds 


\section{Conclusions}

The existence of Negative Differential Resistance in tricyanovinyl-substituted carbazole-based thin-film transistors can be explained by taking into account the geometry of the device and the interaction between Drain-Source and Gate potential. The application of larger drain-source voltage counteracts the electrical field generated by the gate-source voltage near the drain contact, thereby reducing the number of available states in the channel and thus decreasing the current. A correlation has been found between the prevalence of the NDR effect and the length of the tricyanovinyl-substituted carbazole alkyl chain. The amount of NDR can therefore be tuned to the needs of specific applications by changing the length of the aliphatic chain present in the compound.

Acknowledgments: This work was supported by the Spanish Government through

Projects ENE2016-78933-C4-1-R, ENE2017-87671-C3-2-R, and TEC2017-82305-R. J.P personally acknowledges support from Ainet, Eugenia, Quim and Natividad.

\section{References}

[1] H. Sirringhaus, Adv. Mater., 26, 1319-1335 (2014). DOI: 10.1002/adma.201304346

25th Anniversary Article: Organic Field-Effect Transistors: The Path Beyond Amorphous Silicon.

[2] A. Nathan, A. Ahnood.

Proc. the IEEE 100, 1468 (2012). DOI: 10.1109/JPROC.2012.2190168

Flexible electronics: the next ubiquitous platform.

[3] Y.H.Lee, M. Jang, M.Y.Lee, O.Y. Kweon, J.H. Oh.

Chem 3, pp. 724-763, (2017). DOI: 10.1016/j.chempr.2017.10.005

Flexible Field-Effect Transistor-Type Sensors Based on Conjugated Molecules

[4] J.S. Chang, A.F. Faccchetti, R. Reuss.

IEEE Journal on Emerging and Selected Topics in Circuits and Systems, 7 (1), pp. 7-26, (2017) DOI: $10.1109 /$ JETCAS.2017.2673863

A Circuits and Systems Perspective of Organic/Printed Electronics: Review, Challenges, and Contemporary and Emerging Design Approaches

[5] C. Liao, M. Zhang, M.Y. Yao, T.Hua. L. Li.F. Yan

Adv. Mat. Vol. 27 (46) pp. $7493-7527$ (2015). DOI:10.1002/adma.201402625

Flexible Organic Electronics in Biology: Materials and Devices

[6] Organic and Printed Electronics - Fundamentals and Applications

Edited by G. Nisato, D. Lupo, S. Ganz. Chapter 5. Pan Stanford Publishing Pte. Ltd. (2016)

ISBN: 9789814669740 
[7] H. H. Choi, K. Cho, C. D. Frisbie, H. Sirringhaus, V. Podzorov, Nat. Mater., 17(2), pp. 2-7 (2018). DOI: 10.1038/nmat5035

Critical assessment of charge mobility extraction in FETs

[8] H. Phan, M. J. Ford, A. T. Lill, M. Wang, G. C. Bazan, T.-Q. Nguyen

Adv. Funct. Mater. 1707221 (2018). DOI:10.1002/adfm.201707221

Electrical Double-Slope Nonideality in Organic Field-Effect Transistors.

[9] E.G. Bittle, J.I. Basham, T.N. Jackson, O.D. Jurchescu, D.J. Gundlach

Nat. Commun. 7:10908 (2016). DOI: 10.1038/ncomms10908

Mobility overestimation due to gated contacts in organic field-effect transistors

[10] M. Mahdouani, W. Boukhili, R. Bourguiga

Materials Today Communications, 13, pp. 367-377 (2017) DOI: 10.1016/j.mtcomm.2017.11.005

Negative output differential resistance effect in organic thin film transistors based on pentacene:

Characterization and modeling

[11] C. Voz, A. Marsal, C. Moreno, J. Puigdollers, R. Alcubilla

Synthetic metals, 161 (23-24), pp. 2554 - 2557 (2011). DOI: 10.1016/j.synthmet.2011.10.009

Comparison between the density-of-states of picene transistors measured in air and under vacuum

[12 ] R. Tsu and L. Esaki,

Tunneling in a finite superlattice

Appl. Phys. Lett. 22, 562 (1973). DOI: 10.1063/1.1654509

[13] S. J. Wei and H. C. Lin,

Multivalued SRAM Cell Using Resonant Tunneling Diodes

IEEE J. Solid-State Circuits, 27, pp. 212-216 (1992). DOI: 10.1109/4.127345

[14] S. Bhattacharyya, S. J. Henley, E. Mendoza, L. Gomez-Rojas, J. Allam and S. R. P. Silva, Resonant tunnelling and fast switching in amorphous-carbon quantum-well structures

Nat. Mater., 5, 19-22 (2006). DOI: 10.1038/nmat1551

[15] M. Reig, J. Puigdollers, D. Velasco

Solid-state organization of n-type carbazole-based semiconductors for organic thin-film transistors

Phys. Chem. Chem. Phys., 20 (2), pp. 1142-1149 (2018). DOI: 10.1039/C7CP05135B

[16] S. Kobayashi, T. Nishikawa, T. Takenobu, S. Mori, T. Shimoda, T. Mitani, H. Shimotani, N. Yoshimoto, S. Ogawa, Y. Iwasa.

Nat Mater., 3(5), 317-322 (2004). DOI: 10.1038/nmat1105

Control of carrier density by self-assembled monolayers in organic field-effect transistors.

[17] S.M. Sze, K.K. Ng

Physics of Semiconductor Devices, 3rd ed.; Wiley-Interscience: Hoboken, 2007.

[18] G. Horowitz.

J. Mater. Res. 19(07), pp. 1946-1962 (2004). DOI: 10.1557/JMR.2004.0266

Organic thin film transistors: from theory to real devices,

[19] Ch.R. Newman, C. D. Frisbie, D.A. da Silva Filho, J-L. Bredas, P.C. Ewbank, K.R. Mann Chem. Mater., 16, 4436-4451 (2004). DOI: 10.1021/cm049391x

Introduction to Organic Thin Film Transistors and Design of n-Channel Organic Semiconductors 
[20] S. Datta, Lessons from Nanoelectronics: A New Perspective on Transport (Singapore: World Scientific Publishing Company, Singapore, 2012).

[21] S. Datta, Electronic Transport in Mesoscopic Systems (Cambridge University Press, Cambridge, England, 1995).

[22] D. Cahen and A. Kahn.

Adv. Mater., 15, 271 (2003). DOI: 10.1002/adma.200390065

Electron Energetics at Surfaces and Interfaces: Concepts and Experiments

[23] J.C. Blakesley and D. Neher.

Phys. Rev. B84, 075210 (2011) DOI:10.1103/PhysRevB.84.075210

Relationship between energetic disorder and open-circuit voltage in bulk heterojunction organic solar cells.

[24] R.Häusermann, K.Willa, B.Blülle, T.Morf, A. Facchetti, Z. Chen, J. Leed, B.Batlogg Org. Electron., 28, 306-313 (2016). DOI: 10.1016/j.orgel.2015.11.011

Device performance and density of trap states of organic and inorganic field-effect transistors

[25] H.M. Heitzer, T.J. Marks, M.A. Ratner.

J. Am. Chem. Soc., 137 (22), 7189-7196 (2015). DOI: 10.1021/jacs.5b03301

Molecular Donor-Bridge-Acceptor Strategies for High-Capacitance Organic Dielectric Materials

[26] Kevin F. Brennan, "The physics of semiconductors with applications to optoelectronic devices", Cambridge University Press (1999) 


\section{Supplementary information}

\section{Complementing mobility:}

When fitting the fabricated output characteristics, we observed an underestimation of the current in the linear regime while at saturation the equation displayed a perfect matching as seen in Figure 9. We think that this is an indicative of several effects causing an increase in the degree of Negative Differential Resistance.

From the geometric distribution of the electrodes the assumption that the Drain Source voltage relocates the Density of states in the opposite direction of the Gate potential not only affects to the number of available states in the channel (i.e. available states for hopping) but it also affects the field effect mobility. It has already been reported how the mobility can be enhanced by means of the gate potential. From the energetic view of a TFT we can understand that $\mathrm{V}_{\mathrm{GS}}$ is displacing the DOS in a regime where there are states available for conduction between the source and drain fermi levels (i.e. past the threshold voltage), as $V_{D S}$ increases we start to see the interacting effect between $V_{D S}$ and $V_{G S}$ and this DOS moves in the opposite direction to the $V_{G S}$ therefore decreasing the density of states available for conduction. In NDR devices such an effect could be spotted in the output characteristics as the less energetic states that contribute to the hopping of the current are traps with a constrained mobility as seen in Figure 11. Particularly for our fittings the saturation mobility extracted from the transfer fits perfectly the curve in saturation regime Figure 9 therefore we think this could be due to a transition of mobility within the drain source voltage swept. Assuming a lineal decrease of the mobility before reaching saturation value as seen in Figure 10 for the different compounds there is a perfect fitting of the experimental in the linear regime previously presented in Figure 8. The change in mobility assumed is minimal however as the mobility appears also in the denominator of the NDR output equation this greatly affects the output. We believe that a further step in this model could be the use of a smooth transition of the mobility mobilities perhaps using a quadratic form instead of a linear decay which could better describe this phenomenon represented in Figure 10 with the guide to the eye. 

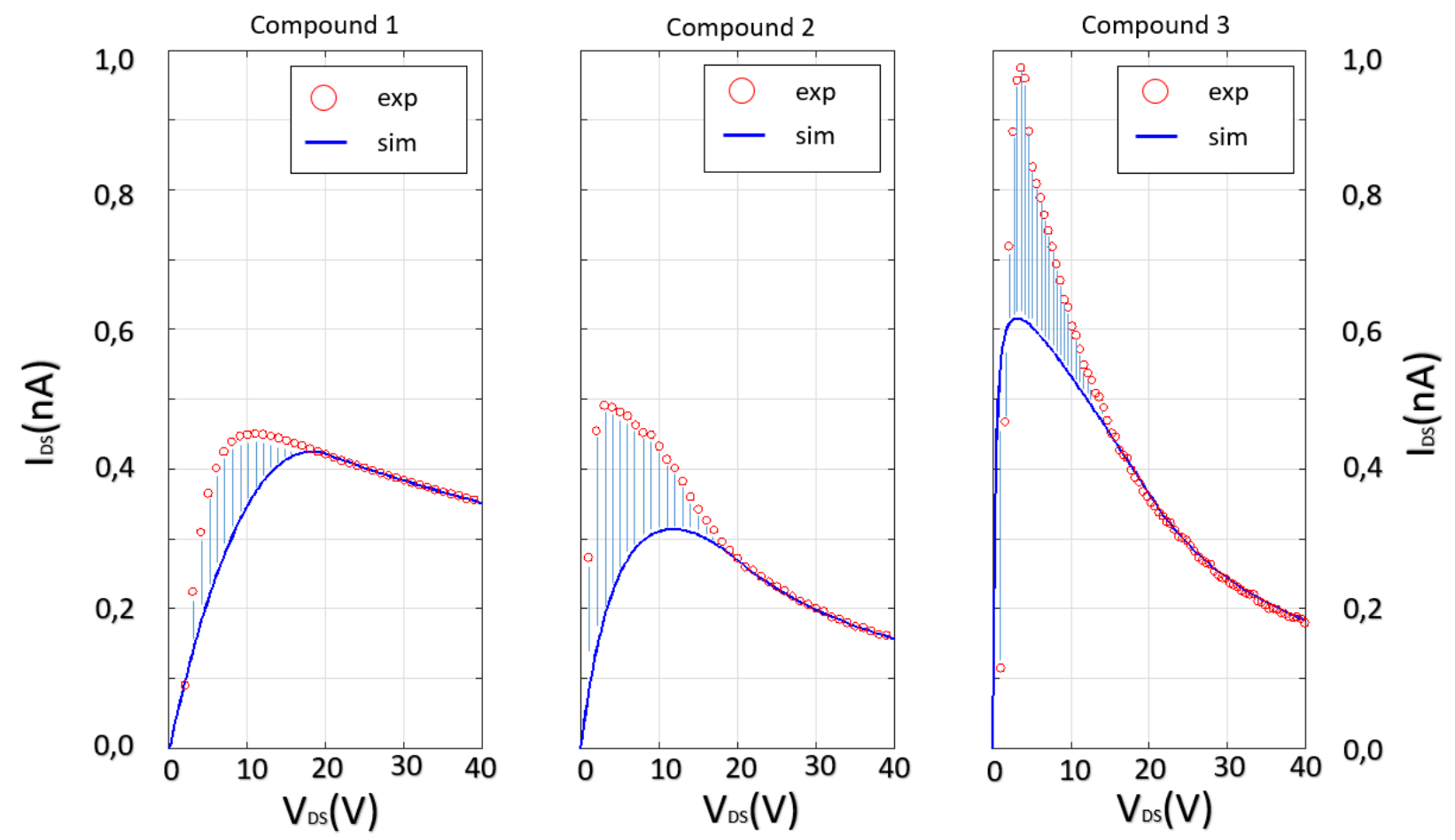

Figure 9

Output characteristics obtained using equation (8) and a constant saturation mobility

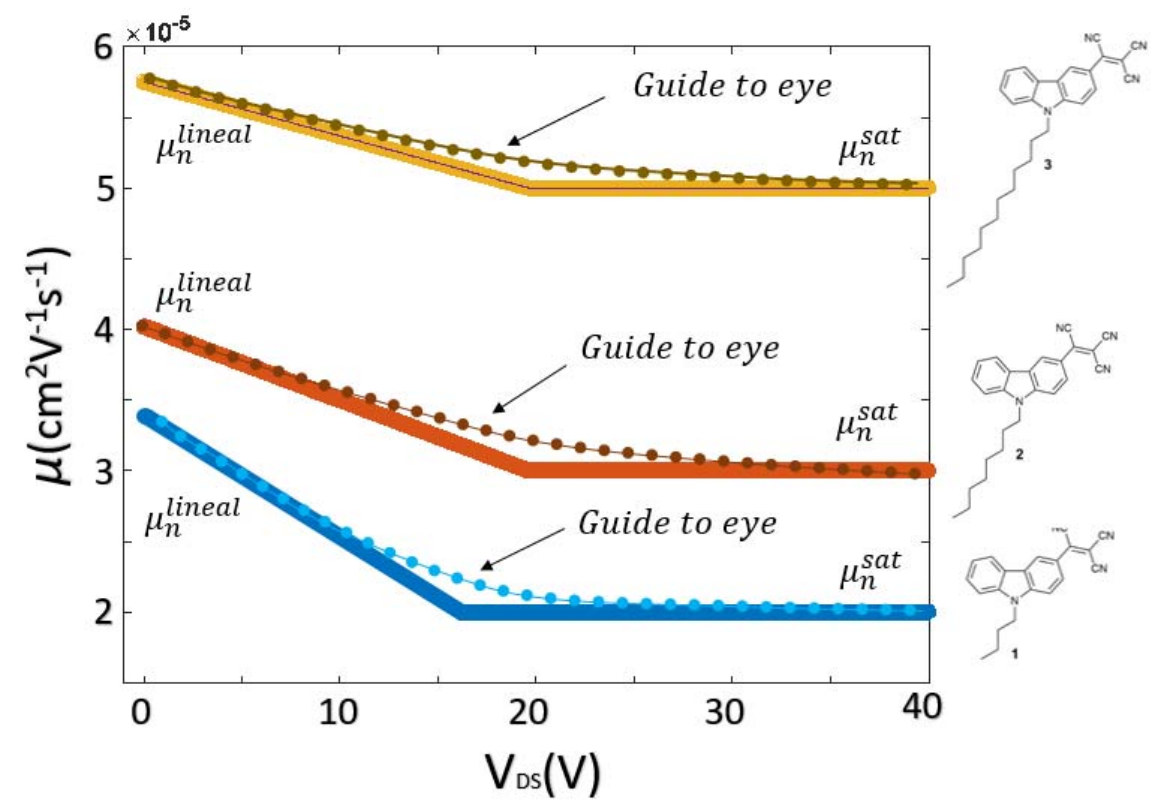

Figure 10

First order change on the mobility as the device enters in the saturation regime 


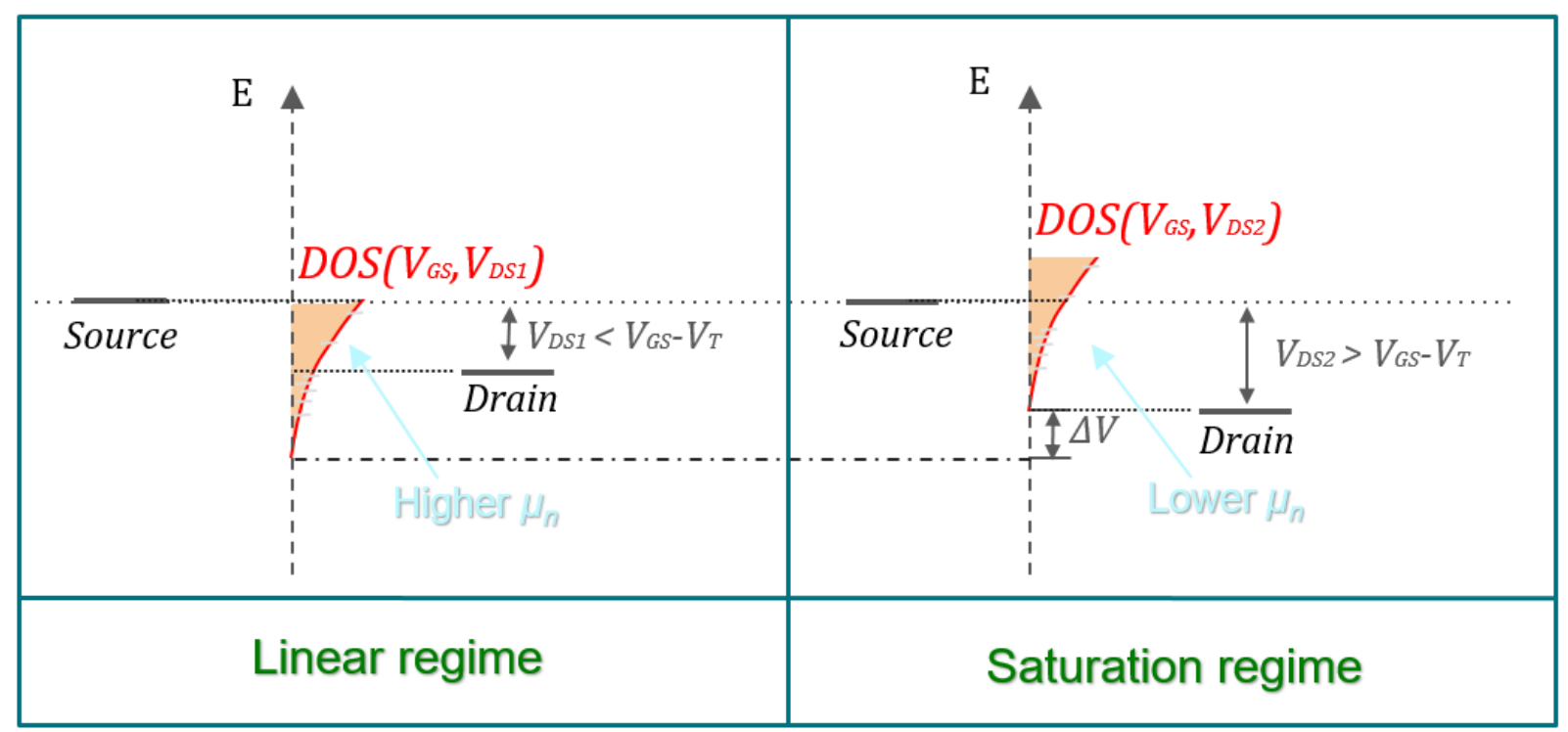

Figure 11:

Schematic of lower mobility states and traps becoming active in the conduction of the channel as the OTFT enters the saturation regime. 
The static dielectric constants $\varepsilon$ (permittivity) of the compounds is estimated according to the Clausius-Mossotti equation:

$$
\varepsilon=\left(1+\frac{8 \pi \alpha}{3 V}\right) \times\left(1-\frac{4 \pi \alpha}{3 V}\right)^{-1}
$$

where $\alpha$ is an theoretical estimated isotropic polarizability, $\mathrm{V}$ is a molecular volume plus the Van der Waals radius of $1 \AA$. The isotropic polarizabilities was estimated by using semi-local hybrid functional B3LYP and 6-31G(d,p) basis set of density function theory (DFT)(1). The molecular structures of the compounds were optimized via B3LYP/6-31G(d,p) level of DFT theory by employing the coordinates from single crystal a nalysis of corresponding compounds as a starting point.

[1] A. Natan, N. Kuritz, L. Kronik. Avd. Func. Mat, Vol.20 (13), 2077-2084 (2010). DOI: $10.1002 /$ adfm.200902162 